\section{Protecting the nation's most valuable resource}

When a nation's young people are in trouble, so is the future of that nation. Given the current state of mental health among children younger than age 18 in the United States, the future of our country looks a bit shaky. The most conservative figures estimate $12 \%$ of children in this age group have a mental disorder. Nearly $50 \%$ of these approximately 7.5 million youngsters are severely mentally handicapped. Yet, only one child in five receives treatment. Most often, the family physician provides this first line of medical care.

Because osteopathic physicians make up $20 \%$ of all family physicians in this country, we have a significant opportunity to provide such first-line treatment. Certainly, we are not all trained psychiatrists. Thus, the National Institute of Mental Health (NIMH) has developed programs to help all nonpsychiatristphysicians recognize the signs and symptoms of commonly missed mental disorders.

Part of this educational process involves understanding the multidimensional facets of mental health disorders. To that end, the $\mathrm{Na}-$ tional Advisory Mental Health Council, under the auspices of NIMH, prepared the report National Plan for Research on Child and Adolescent Mental Disorders, which was recently submitted to Congress.

Each year, mental disorders among young people cost the healthcare system more than $\$ 1.5$ billion dollars. (No monetary value can be placed on the emotional pain patients and their families experience.) Substance abuse often accompanies mental health disorders, making medical treatment more complex and costly.

Genetic factors have also been implicated in mental disorders such as schizophrenia, manic-depressive illness, autism, Tourette's syndrome, obsessive-compulsive disorder, and certain learning disabilities, such as dyslexia. By applying rapidly advancing genetic research techniques to existing knowledge, researchers may be able to identify and intervene in genetically vulnerable children before such disorders develop.

Likewise, advances in imaging techniquesPET, MRI, BEAM, SPECT-enable researchers to safely examine the brain's structure and electric and metabolic activity. In fact, specific patterns of brain pathology in autism and schizophrenia have been identified using these noninvasive imaging modalities. Further knowledge gained using these and other techniques will not only enhance diagnostic precision but suggest ways to correct abnormal brain function. In this way, severe, chronic mental disorders may be cured or at least controlled.

The report also addresses the influence persistent psychosocial adversity has on the development of mental disorders. Some children subjected to poverty, abuse, or neglect, and disturbed family relationships have an increased risk of developing mental disorders. Yet, some youngsters who are from extremely adverse environments develop well; apparently, protective factors naturally shield them from developmental harm. Further studies in this area are expected to yield methods that will strengthen these protective factors. Perhaps mental illness could then be prevented in environmentally threatened children. Increasing our understanding of the interaction between such biopsychosocial factors will lead to methods that could prevent impairment when applied early in a child's cognitive and socialemotional development.

Continued progress in defining and identifying discreet mental disorders in young people depends on several factors. The NIMH hopes to stimulate-and provide the leadership and coordination of-wide-ranging basic clinical research. In fact, the stage has already (continued on page 681) 
Daingisol Hypertensive
and overweight

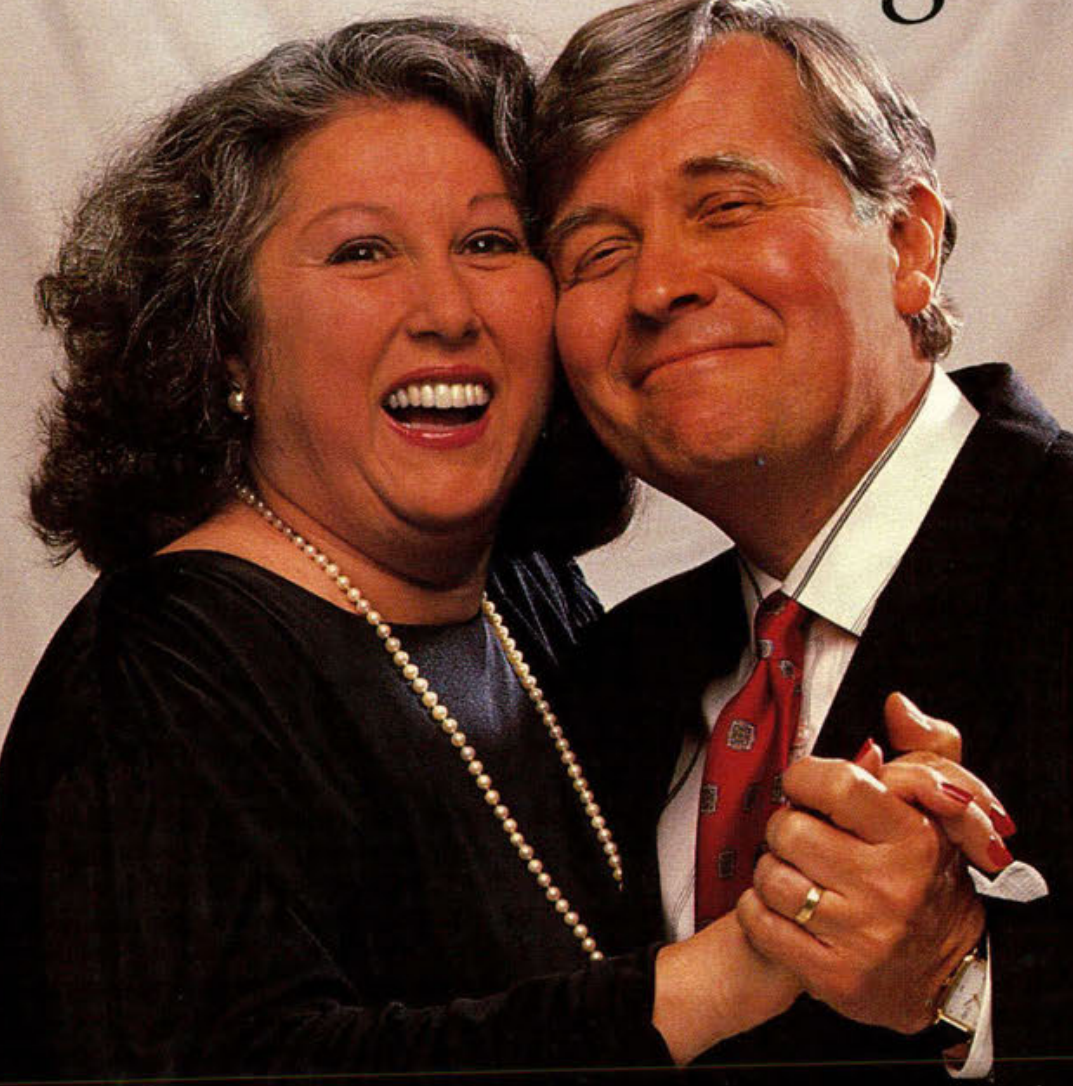

CAPOTEN, for your hypertensive patients, including the overweight. Prescribe Capoten 25 mg bid.*

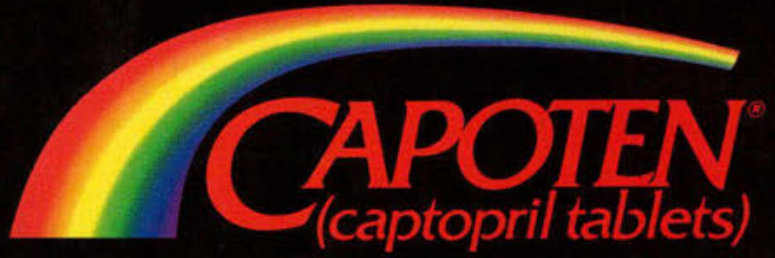

Bring them under the rainbow.

- CAPOTEN may be used as initial therapy only for patients with normal renal function in whom the risk of neutropenia/agranulocytosis is relatively low (1 out of over 8,600 in clinical trials). Use special precautions in patients with impaired renal function, collagen vascular disorders, or those exposed to other drugs known to affect the white blood cells or immune response. Evaluation of hypertensives should always include assessment of renal function. See INDICATIONS AND USAGE, CONTRAINDICATIONS, WARNINGS, PRECAUTIONS, and ADVERSE REACTIONS in the brief summary of prescribing information on the next page. 


\section{CAPOTEN* TABLETS}

Captopril Tablets

INDICATIONS: Hypertension-CAPOTEN (captopril) is indicated for the treatment of hypertension. Consideration should be given to the risk of neutropenia/ agranulocytosis (see WARNINGS). CAPOTEN is effective alone and in combination with other antihypertensive agents, especially thiazide-type diuretics.

Heart Failure: CAPOTEN (captopril) is indicated in the treatment of congestive heart failure in patients who have not responded adequately to treatment with diuretics and digitalis. poorly tolerated or otherwise not feasible.

CONTRAINDICATIONS: CAPOTEN is contraindicated in patients who are hypersensitive to this product

WARNINGS: Angioedema-Angioedema involving the extremities, face, lips, mucous membranes, tongue, glottis or larynx has been seen in patients treated with ACE inhibitors, including captopril. If angioedema involves the tongue, glottis or larynx, airway obstruction may occur and be fatal. Emergency therapy, including but not necessarily limited to, subcutaneous administration of a 1:1000 solution of epinephrine should be promptly instituted.

Neutropenia/Agranulocytosis - Neutropenia $\left(<1000 / \mathrm{mm}^{3}\right)$ with myeloid hypoplasia has resulted from resulted from use of captopril. About half of the neutropenic patients developed systemic or oral cavity infections or other features of the syndrome of
neutropenia is dependent on the clinical status of the patient:

In clinical trials in patients with hypertension who have normal renal function (serum creatinine less than $1.6 \mathrm{mg} / \mathrm{dL}$ and no collagen vascular disease), neutropenia has been seen in one patient out of over 8.600 exposed. In patients with some degree of renal failure (serum creatinine at least $1.6 \mathrm{mg} / \mathrm{dL}$ ) but no collagen vascular disease, the risk in clinical trials was about 1 per 500 . Doses were relatively high in these patients, particularly in view of their diminished renal function. In patients with collagen vascular diseases (e.g., systemic lupus erythematosus, scleroderma) and impaired renal function, neutropenia occurred in $3.7 \%$ of patients in clinical trials. While none of the over 750 patients in formal clinical trials of heart failure developed neutropenia, it has occurred during the subsequent clinical experience. Of reported cases, about half had serum creatinine $>1.6 \mathrm{mg} / \mathrm{dL}$ and more than $75 \%$ received procainamide. In heart failure, it appears that the same risk factors for neutropenia are present.

Neutropenia has appeared usually within 3 months after starting therapy, associated with myeloid hypoplasia and frequently accompanied by erythroid hypoplasia and decreased numbers of megakaryocytes (e.g., hypoplastic bone marrow and pancytopenia); anemia and thrombocytopenia were sometimes seen. Neutrophils generally returned to normal in about 2 weeks after captopril was discontinued, and serious infections were limited to clinically complex patients. About $13 \%$ of the cases of neutropenia have ended fatally, but almost all fatalities were in patients with serious illness, having collagen vascular disease, renal failure, heart failure or immunosuppressant therapy, or a combination of these complicating factors. Evaluation of the hypertensive or heart failure patient should always include assessment of renal function. If captopril is used in patients with impaired renal function, white blood cell and differential counts should be evaluated prior to starting treatment and at approximately 2 -week intervals for about 3 months, then periodically. In patients with collagen vascular disease or who are exposed to other drugs known to affect the white cells or immune response, particularly when there is impaired renal function, captopril should be used only after an assessment of benefit and risk, and then with caution. All patients treated with captopril should be told to report any signs of infection (e.g. sore throat, fever). If infection is suspected, perform white cell counts without delay Since discontinuation of captopril and other drugs has generally led to prompt return of the white count to normal, upon confirmation of neutropenia (neutrophil count $<1000 / \mathrm{mm}^{3}$ ) withdraw captopril and closely follow mation of neutropen

Proteinuria: Total urinary proteins $>1 \mathrm{~g}$ per day were seen in about $0.7 \%$ of patients on captopril. About $90 \%$ of affected patients had evidence of prior renal disease or received high doses ( $150 \mathrm{mg} /$ day), or both. The nephrotic syndrome occurred in about one-fifth of proteinuric patients. In most cases, proteinuria subsided or cleared within 6 months whether or not captopril was continued. The BUN and creatinine were seldom altered in proteinuric patients. Since most cases of proteinuria occurred by the 8 th month of therapy with captopril, patients with prior renal disease or those receiving captopril at doses $150 \mathrm{mg}$ per day, should have urinary protein estimates (dip-stick on 1st morning urine) before therapy. and periodically thereafter.

Hypotension: Excessive hypotension was rarely seen in hypertensive patients but is a possibility in severely salt/volume-depleted persons such as those treated vigorously with diuretics (see PRECAUTIONS [Drug Interactions]). In heart failure, where the blood pressure was either normal or low, transient decreases in mean blood pressure $20 \%$ were recorded in about half of the patients. This transient hypotension may occur after any of the first several doses and is usually well tolerated, although rarely it has been associated with arrhythmia or conduction defects. A starting dose of 6.25 or $12.5 \mathrm{mg}$ tid may minimize the hypotensive effect. Patients should be followed closely for the first 2 weeks of treatment and whenever the dose of captopril and/or diuretic is increased.

BECAUSE OF THE POTENTIAL FALL IN BLOOD PRESSURE IN THESE PATIENTS, THERAPY SHOULD BE STARTED UNDER VERY CLOSE MEDICAL SUPERVISION.

PRECAUTIONS: General: Impaired Renal Function-Hypertension-Some hypertensive patients with renal disease, particularly those with severe renal artery stenosis, have developed increases in BUN and serum creatinine. It may be necessary to reduce captopril dosage and/or discontinue diuretic. For some of these patients, normalization of blood pressure and maintenance of adequate renal perfusion may not be possible. Heart Failure-About $20 \%$ o patients develop stable elevations of BUN and serum creatinine $20 \%$ above normal or baseline upon long-term treatment. Less than $5 \%$ of patients, generally with severe preexistin renal disease, required discontinuation due to progressively increasing creatinine. See DOS AGE AND ADMINISTRATION, ADVERSE REACTIONS [Altered Laboratory Findings]. Valvular Stenosis-A theoretical concern, for risk of decreased coronary perfusion, has been noted regarding vasodilator treatment in patients with aortic stenosis due to decreased afterload reduction. Surgery/Anesthesia-If hypotension occurs during surgery or anesthesia, and is reduction. Surgery/Anesthesia-If hypotension occurs during surgery or anesth

Drug Interactions: Hypotension-Patients on Diuretic Therapy-Precipitous reduction of blood pressure may occasionally occur within the 1st hour after administration of the initial captopril dose in patients on diuretics, especially those recently placed on diuretics, and those on severe dietary salt restriction or dialysis. This possibility can be minimized by either discontinuing the diuretic or increasing the salt intake about 1 week prior to initiation of captopril therapy or by initiating therapy with small doses $(6.25$ or $12.5 \mathrm{mg})$. Alternatively, provide medical supervision for at least 1 hour after the initial dose.

Agents Having Vasodilator Activity-In heart failure patients, vasodilators should be ad ministered with caution

Agents Causing Renin Release-Captopril's effect will be augmented by antihypertensive agents that cause renin release.

Agents Affecting Sympathetic Activity-The sympathetic nervous system may be especially important in supporting blood pressure in patients receiving captopril alone or with diuretics. Beta-adrenergic blocking drugs add some further antihypertensive effect to captopril, but the overall response is less than additive. Therefore, use agents affecting sym pathetic activity (e.g. ganglionic blocking agents or adrenergic neuron blocking agents) with pathetic
caution.

(c) 1990 E.R. Squibb \& Sons, Inc., Princeton, NJ
Agents Increasing Serum Potassium-Give potassium-sparing diuretics or potassium supplements only for documented hypokalemia, anche significant increase of serum potassium. Use potassium-containing salt substitutes with caution.

Inhibitors of Endogenous Prostaglandin Synthesis-Indomethacin and other nonsteroidal anti-infiammatory agents may reduce the antihypertensive effect of captopril, especially in low renin hypertension.

Lithium-increased serum lithium levels and symptoms of lithium toxicity have been reported in patients receiving concomitant lithium and ACE inhibitor therapy. These drugs should be coadministered with caution and frequent monitoring of serum lithium levels is recommended If a diuretic is also used, it may increase the risk of lithium toxicity.

Drug/Laboratory Test Interaction: Captopril may cause a false-positive urine test for acetone Carcinogenesis, Mutagenesis and Impairment of Fertility: Two-year studies with doses of 50 to $1350 \mathrm{mg} / \mathrm{kg} /$ day in mice and rats failed to show any evidence of carcinogenic potential. 50 to $1350 \mathrm{mg} / \mathrm{kg} /$ day in mice and rats failed to show an
Studies in rats have revealed no impairment of fertility.

Pregnancy: Category C: Embryocidal effects and craniofacial malformations were observed in rabbits. Human Experience-There are no adequate and well-controlled studies of captopril in pregnant women. Data are available that show captopril crosses the human placenta Captopril should be used during pregnancy only if the potential benefit justifies the potential risk to the fetus.

Based on post-marketing experience with all ACE inhibitors, the following information has been collected. Inadvertent exposure limited to the first trimester of pregnancy does no appear to affect fetal outcome adversely. Fetal exposure during the second and third trimester of pregnancy has been associated with fetal and neonatal morbidity and mortality.

When ACE inhibitors are used during the later stages of pregnancy, there have been reports of hypotension and decreased renal perfusion in the newborn. Oligohydramnios in the mother has also been reported. Infants exposed in utero to ACE inhibitors should be closely observed for hypotension, oliguria and hyperkalemia. If oliguria occurs, attention should be directed toward support of blood pressure and renal perfusion with the administration of fluids and pressors as appropriate. Problems associated with prematurity such as patent ductus arteriosus have occurred in association with maternal use of ACE inhibitors but it is not clear whether they are related to ACE inhibition, maternal hypertension or the underlying prematurity

There is no experience with exchange transfusion, hemodialysis or peritoneal dialysis for removing captopril from the neonatal circulation

Nursing Mothers: Captopril is secreted in human milk. Exercise caution when administering captopril to a nursing woman, and, in general, nursing should be interrupted.

Pediatric Use: Safety and effectiveness in children have not been established.

ADVERSE REACTIONS: Reported incidences are based on clinical trials involving approximately 7000 patients

Renal-About 1 of 100 patients developed proteinuria (see WARNINGS). Renal insufficiency. enal failure, polyuria, oliguria, and urinary frequency in 1 to 2 of 1000 patients.

Hematologic-Neutropenia/agranulocytosis has occurred (see WARNINGS). Anemia hrombocytopenia, and pancytopenia have been reported.

Dermatologic-Rash, (usually maculopapular, rarely urticarial), often with pruritus, and sometimes with fever and eosinophilia, in about 4 to 7 of 100 patients (depending on renal status and dose), usually during the 1 st 4 weeks of therapy. Pruritus, without rash, in about 2 of 100 patients. A reversible associated pemphigoid-like lesion, and photosensitivity, have also been reported. Flushing or pallor in 2 to 5 of 1000 patients.

Cardiovascular-Hypotension may occur; see WARNINGS and PRECAUTIONS [Drug Interactions] for discussion of hypotension on initiation of captopril therapy. Tachycardia chest pain, and palpitations each in about 1 of 100 patients. Angina pectoris, myocardial infarction, Raynaud's syndrome, and congestive heart failure each in 2 to 3 of 1000 patients. Dysgeusia-Approximately 2 to 4 (depending on renal status and dose) of 100 patients developed a diminution or loss of taste perception; taste impairment is reversible and usually self-limited even with continued drug use ( 2 to 3 months).

Angioedema-Angioedema involving the extremities, face, lips, mucous membranes, tongue, glottis or larynx has been reported in approximately one in 1000 patients. Angioedema involving the upper airways has caused fatal airway obstruction. (See WARNINGS.)

The following have been reported in about 0.5 to 2 percent of patients but did not appear at increased frequency compared to placebo or other treatments used in controlled trials: gastric irritation, abdominal pain, nausea, vomiting, diarrhea, anorexia, constipation, aphthous ulcers, peptic ulcer, dizziness, headache, malaise, fatigue, insomnia, dry mouth, dyspnea, cough, alopecia, paresthesias.

Other clinical adverse effects reported since the drug was marketed are listed below by body system. In this setting. an incidence or causal relationship cannot be accurately determined.

General: Asthenia, gynecomastia

Cardiovascular: Cardiac arrest, cerebrovascular accident, syncope.

Dermatologic: Bullous pemphigus.

Gastrointestinal: Pancreatitis, glossitis.

Hematologic: Anemia, including aplastic and hemolytic

Hepatobiliary: Hepatitis, including rare cases of necrosis, cholestasis

Metabolic: Symptomatic hyponatremia.

Nervous/Psychiatric: Ataxia, confusion, depression, nervousness, somnolence.

Respiratory: Bronchospasm, eosinophilic pneumonitis, rhinitis

Special Senses: Blurred vision

Urogenital: Impotence.

As with other ACE inhibitors, a syndrome has been reported which includes: fever, myalgia, arthralgia, rash or other dermatologic manifestations, eosinophilia and an elevated ESR. Findings have usually resolved with discontinuation of treatment

Altered Laboratory Findings: Serum Electrolytes: Hyperkalemia: small increases in serum potassium, especially in patients with renal impairment (see PRECAUTIONS).

Hyponatremia: particularly in patients receiving a low sodium diet or concomitant diuretics BUN/Serum Creatinine: Transient elevations of BUN or serum creatinine especially in volume or salt depleted patients or those with renovascular hypertension may occur. Rapid reduction of longstanding or markedly elevated blood pressure can result in decreases in the glomerular filtration rate and, in turn, lead to increases in BUN or serum creatinine.

Hematologic: A positive ANA has been reported.

Liver Function Tests: Elevations of liver transaminases, alkaline phosphatase, and serum bilirubin have occurred.

OVERDOSAGE: Primary concern is correction of hypotension. Volume expansion with an I.V. infusion of normal saline is the treatment of choice for restoration of blood pressure. Captopril may be removed from the general circulation by hemodialysis.

DOSAGE AND ADMINISTRATION: CAPOTEN (captopril) should be taken one hour before meals. In hypertension, CAPOTEN may be dosed bid or tid. Dosage must be individualized; see DOSAGE AND ADMINISTRATION section of package insert for detailed information regarding dosage in hypertension and in heart failure. Because CAPOTEN (captopril) is excreted primarily by the kidneys, dosage adjustments are recommended for patients with impaired renal function.

Consult package insert before prescribing CAPOTEN (captopril).

HOW SUPPLIED: Available in tablets of 12.5, 25, and $50 \mathrm{mg}$ in bottles of 100 and 1000; 100 $\mathrm{mg}$ in bottles of 100; and in UNIMATIC" unit-dose packs of 100 tablets.

(J3-658R) 